\title{
RESEARCH HIGHLIGHT Heart defects recapitulated in human cardioids
}

Valeria V. Orlova $\mathbb{D}^{1}$ and Christine L. Mummery $\mathbb{D}^{1 凶}$

(c) CEMCS, CAS 2021

Cell Research (2021) 31:947-948; https://doi.org/10.1038/s41422-021-00534-5

\begin{abstract}
A major obstacle to understanding how and why congenital defects occur in the heart is the absence of models that recapitulate the basic cell lineage architecture of human heart development. In a recent study in Cell, Hofbauer and colleagues present compelling evidence that they have created such a model from human pluripotent stem cells by guiding selforganization within cell aggregates (or organoids) which they refer to as "cardioids"; these undergo patterning and morphogenesis to form a cavity-like chamber as in early heart development.
\end{abstract}

The last decade has witnessed a tsunami of research on organoids, a term that now alludes to organ-like structures that form spontaneously through self-organization based on developmental mechanisms or are constructed using "tissue engineering" methods. The latter usually contain pre-differentiated cells assembled with or without an extracellular matrix as a carrier, whilst the former contain multiple cell types that retain or establish the identity of the modeled organ, organized in much the same way as in the organ itself. Organoids representative of many organ types have been derived, some based on human adult stem cells from healthy or tumor tissue, and others on human pluripotent stem cells (hPSCs) from embryos or induced by reprogramming of somatic cells. For the heart, however, this has been more challenging, since it seems unlikely that the adult heart contains stem cells that could form organoids and in models based on directed differentiation of hPSCs in cardiogenesis, the presence of (non-cardiac) cells has always been noted. The need for models of the early human heart are clear since congenital heart defects are the most common organ malformation at birth.

Development of the human heart begins just two weeks after fertilization with beating evident at around three weeks. By the fourth week, the least accessible period in development, a four-chambered heart has formed. It is then that congenital heart defects first start, eventually occurring in about $1 \%$ of live births. Among these, hypoplastic left heart syndrome (HLHS) and left ventricular noncompaction of the myocardium (LVNC) are disorders of cardiac chamber maturation: the heart chambers become dilated or hypertrophic. ${ }^{1}$ However, limited understanding of early human development means that the cellular origin and genetic basis of these defects has remained elusive. In fact, many of the gene mutations in cardiac transcription factors or sarcomeric proteins are associated with dilated and hypertrophic cardiomyopathies and are not unique to HLHS and LVNC. Recent studies suggest that HLH and LVNC can also be caused by defective function of the endocardium, the specialized endothelium that forms the inner lining of the heart chambers, or the epicardium, the outer covering of the developing heart. ${ }^{2-4}$

Methods to differentiate hPSCs into all cardiomyocyte subtypes (the contractile heart cells or the pacemaker cells controlling heart rate) as well as endocardial and epicardial cells have now been described ${ }^{5-8}$ presenting new opportunities to understand how heart defects develop. By co-culturing cardiomyocytes with either hPSC-derived endocardial cells or adding factors derived from epicardial cells, for example, features of trabecular or compacted myocardium have been observed. ${ }^{7,9}$ None of these have yet captured the self-organization of heart cells that occurs in development.

In the study published in Cell, ${ }^{10}$ the authors derived highly organized heart-like structures that were devoid of cell types from other lineages like endoderm. Remarkably, they even observed cavity formation, with an inner lining of endocardium, surrounded by a thick layer of myocardium and a covering of epicardial-like cells. This showed that the mesoderm lineage has an intrinsic ability to self-organize and does not require signals from adjacent endoderm also present during development and previously indicated as an essential signaling source for heart development. Detailed analysis of gene expression and cell function showed that the cardioids resembled the chambers of the heart in early development. Perhaps as remarkably, the authors also showed that after cryo-injury, in vivo-like fibronectin and collagen accumulation is initiated, reminiscent of fibrosis following myocardial infarction.

How did they achieve this precise and reproducible recapitulation of early heart development? Essential was their use of decades of research on heart development in model organisms like mouse and zebrafish. Crucial was their identification of the role of WNT and bone morphogenetic protein (BMP) in directing cavity formation via the transcription factor HAND1, aside from the known role of these factors in the differentiation process itself. Notably, high concentrations of WNT during mesoderm induction were necessary for cavity expansion but this was at the expense of cardiomyocyte differentiation. Intermediate levels of WNT were then identified as optimal but this was hPSC line dependent. WNT inhibition was also needed later for optimal cardiomyocyte differentiation, although not for cavity formation as such. BMP inhibition impaired cavity formation and thus was also necessary. Interestingly, low concentrations of WNT and ACTIVIN (that induces NODAL signaling) upregulated expression of endogenous vascular endothelial growth factor (VEGF-A) in cardiogenic mesoderm. This was sufficient to induce formation of vascular endothelial cells that lined the chamber cavity, resembling endocardium in the heart. Finally, cardioids supported epicardial

${ }^{1}$ Department of Anatomy and Embryology, Leiden University Medical Center, Leiden, the Netherlands. ${ }^{凶}$ email: c.l.mummery@lumc.nl 
Hypoplastic left heart

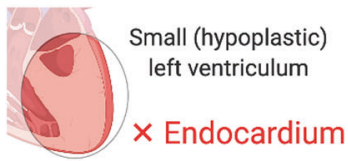

Left ventricular

noncompaction

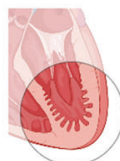

Excessive

trabeculations

$\times$ Endocardium $\times$ Epicardium
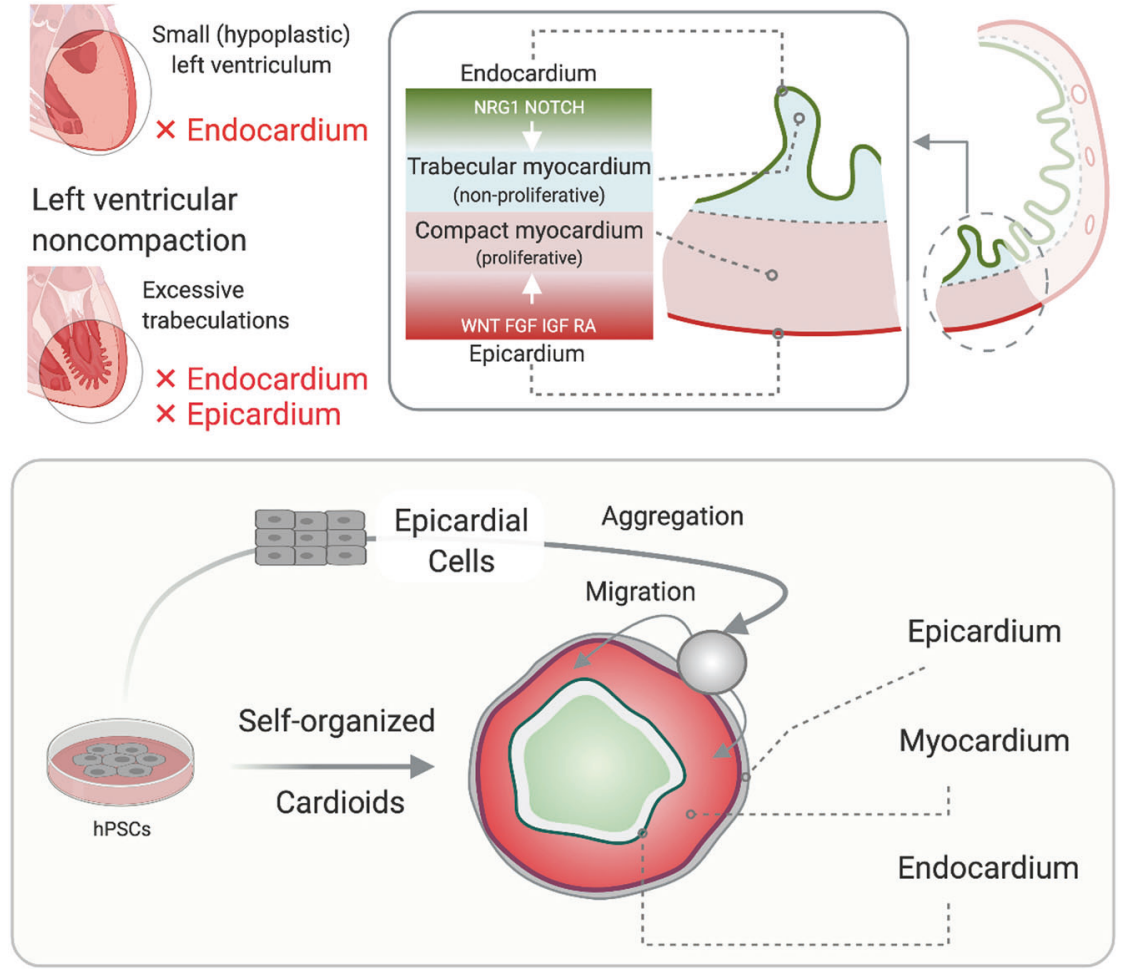

Fig. 1 Human cardioid model to study congenital heart defects. Top: HLHS and left LVNC are disorders of cardiac chamber maturation. Recent studies suggest that HLHS and LVNC can also be caused by defective function of the endocardium or the epicardium. Bottom: Human cardioids are formed by self-organization of cells within cell aggregates and contain endocardium, myocardium, and epicardium, and thus can be used to study congenital defects in human. RA, retinoic acid; FGF, fibroblast growth factor; IGF, insulin-like growth factor. This figure is created with BioRender.com.

cell spreading and migration, much as in the early heart development. The cardioid model thus recapitulates stages of human heart development normally only accessible in animal model organisms.

What could the cardioid model tell us about congenital heart defects? For instance, in HLHS, disrupted levels of the BMPregulated genes NKX2.5 and HAND1 are associated with the severely reduced cardiac cavity in the left ventricular chamber. The hPSC model allowed the authors to delete either the NKX2.5 or HAND1 genes and then form cardioids. Whilst the net result in both cases was that the cardioids were smaller and cavity size was reduced, careful analysis of the timing allowed them to identify exactly which cell types and morphogenetic processes were affected and when. It would be straightforward to use cardioids for other gene mutations associated with congenital defects such as GATA4 and TBX5. This illustrates the potential not only for studying this type of defect but many others, at least those of genetic origin (Fig. 1).

Whilst cardioids are unlikely to replace existing cardiac models mentioned earlier, they comprise a significant advance in cardiac organoid technology, akin to what has been achieved for other organs.

\section{REFERENCES}

1. Rufaihah, A. J., Chen, C. K., Yap, C. H. \& Mattar, C. N. Z. Dis. Model Mech. 14, dmm047522 (2021).

2. Luxán, G., D’Amato, G., MacGrogan, D. \& de la Pompa, J. L. Circ. Res. 118, e1-e18 (2015).

3. Gittenberger-de Groot, A. C. et al. Differentiation 84, 41-53 (2012).

4. Miao, Y. et al. Cell Stem Cell 27, 574-589.e8 (2020).

5. Guadix, J. A. et al. Stem Cell Reports 9, 1754-1764 (2017).

6. Giacomelli, E. et al. Cell Stem Cell 26, 862-879.e11 (2020).

7. Mikryukov, A. A. et al. Cell Stem Cell 28, 96-111 (2021).

8. Protze, S. I., Lee, J. H. \& Keller, G. M. Cell Stem Cell 25, 311-327 (2019).

9. Funakoshi, S. et al. Nat. Commun. 12, 3155 (2021)

10. Hofbauer, P. et al. Cell 184, 3299-3317 (2021).

\section{COMPETING INTERESTS}

C.L.M. is co-founder of Ncardia bv.

\section{ADDITIONAL INFORMATION}

Correspondence and requests for materials should be addressed to C.L.M.

Reprints and permission information is available at http://www.nature.com/ reprints 